\title{
Stage IIIC1
}

National Cancer Institute

\section{Source}

National Cancer Institute. Stage IIIC1. NCI Thesaurus. Code C95179.

A stage term that applies to uterine corpus cancer and indicates that the tumor is confined to the corpus uteri, or it invades the stromal connective tissue of the cervix but does not extend beyond the uterus, or it involves serosa and/or adnexa, vagina, or parametrial tissue. There is regional lymph node metastasis to the pelvic lymph nodes. 